REVIEW ARTICLE

\title{
Viral Infections after Kidney Transplantation: An Updated Review
}

\author{
Ethem Unal*, Ahmet Topcu, Muhammed Taha Demirpolat and Ömer Faruk Özkan
}

Department of General Surgery, Umraniye Training and Research Hospital, Health Sciences University, Istanbul, Turkey

*Corresponding author: Ethem Unal, M.D, Associate Professor of Surgery, Department of General Surgery, Umraniye Training and Research Hospital, Health Sciences University, Agaoglu My City C2/37, Tavukcuyolu Caddesi, Umraniye, Istanbul 34660, Turkey, Tel: +90-532-258-4917, E-mail: drethemunal@gmail.com

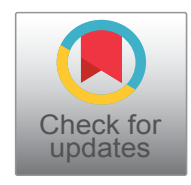

\begin{abstract}
Infections are the major cause of morbidity and mortality in kidney transplant recipients. Careful pretransplant screening, immunization, and posttransplant prophylactic antimicrobials may all reduce the risk for posttransplant infections. Chronic immunosuppression, required to maintain allograft function post-organ transplant, predisposes transplant patients to a variety of viral infections. These can occur at every stage of post-transplantation. Some infections, however, such as cytomegalovirus (CMV), Epstein Barr virus (EBV), or BK virus (BKV), tend to occur within months after transplantation. CMV infections can be easily prevented by prophylaxis therapy, whereas EVB or BKV infections can be prevented by lowering immunosuppression. Some viral infections can result in posttransplant lymphoproliferative disorders (EBV), Kaposi sarcoma (human herpes simplex virus type 8), or skin and/or cervical cancers (papillomavirus). Other viral infections, such influenza viruses, are mostly acquired through environmental spread. These all can be easily detected at early stages, and can be efficiently treated.
\end{abstract}

\section{Keywords}

Viral infection, Kidney transplantation

Despite significant advances in the field of renal transplantation, viral infections continue to be a potential contributor to graft failure, but also a cause of severe mortality and morbidity (Table 1 ). The consequences of viral infections are variable and may include direct involvement of the allograft, dissemination to other end organs, or indirect effects on the patient and allograft [1]. Some viruses, notably herpesviruses and polyomavirus, are thought to further impair host defenses, thereby increasing the risk for other infections [2]. Viral infections have also been implicated as co-factors in acute and chronic rejection syndromes.
CMV is the most common viral infection in solid organ transplant recipients. It usually develops during the first few months after transplantation, and is associated with clinical infectious disease (e.g. fever, pneumonia, gastrointestinal ulcers, hepatitis, retinitis) and acute or chronic graft dysfunction. Sources of CMV infection in transplant recipients include latent reactivation, donor-transmitted virus, and virus present in donor leukocytes. Approximately $20 \%$ to $60 \%$ of all transplant recipients develop symptomatic CMV infection [3]. Most cases of symptomatic CMV infection can be characterized by a self-limiting syndrome of episodic fever attacks for a period of 2 to 4 weeks, arthralgias, fatigue, anorexia, abdominal pain, and diarrhea. Moreover,

Table 1: Most commonly seen viral infections in renal transplant recipients.

\begin{tabular}{|l|}
\hline Cytomegalovirus (CMV) \\
\hline Herpes simplex (HSV) \\
\hline Varicella zoster virus (VZV) \\
\hline Epstein Barr virus (EBV) \\
\hline Polyomaviruses (BK, JC, SV-40) \\
\hline Human herpes virus 6,7,8 (HHV) \\
\hline Human T-lymphotropic virus 1 and 2 (HTLV) \\
\hline Hepatitis B and C viruses (HBV, HCV) \\
\hline Human immunodeficiency virus (HIV) \\
\hline Human papillomavirus (HPV) \\
\hline West Nile virus (WNV) \\
\hline Adenovirus \\
\hline Respiratory syncytial virus (RSV) \\
\hline Influenza and parainfluenza viruses \\
\hline Human metapneumovirus \\
\hline Rhinovirus \\
\hline Coronavirus \\
\hline Rabies virus \\
\hline
\end{tabular}


CMV infection can disseminate to the lungs, liver, pancreas, kidneys, gastrointestinal tract and brain, and can cause death. CMV causes renal allograft injury that may be indistinguishable from injury caused by rejection or other factors, and it has been linked to acute rejection and chronic rejection. Current diagnostic strategies rely on the detection of CMV viremia; antibody testing and culture are less sensitive and delay diagnosis. Viremia may be detected using the CMV antigenemia assay or by nucleic acid testing (NAT). Treatment of established CMV disease requires a multifactorial approach, including reduction of immunosuppressive agents, antiviral agents, and in some cases adjuvant therapy. Intravenous ganciclovir has been considered the mainstay of therapy. Valganciclovir is an oral prodrug of ganciclovir, with a 10-fold greater bioavailability than oral ganciclovir. Most recent studies suggest that valganciclovir has the potential to replace both oral and IV ganciclovir in many situations [4].

HSV and VZV are both alpha herpes viruses with a double stranded DNA core. Infection in the renal transplant patient is usually caused by reactivation of latent virus. HSV infection usually presents with oral or genital lesions, but in some instances can cause esophagitis, hepatitis, encephalitis or pneumonitis $[1,5]$. VZV reactivation usually presents as dermatomal zoster, but can disseminate, causing similar visceral complications. In the absence of prophylaxis, HSV and VZV may be seen early with HSV observed even in the first post transplant month and VZV as early as 1 to 6 mo post transplant. Post-transplant prophylaxis against reactivation of VZV and HSV is recommended to prevent severe recurrences and consists of ganciclovir in patients needing CMV prophylaxis [5]. Those patients who do not require CMV prophylaxis, can receive valacyclovir or acyclovir for approximately one to three months post transplant $[5,6]$. Diagnosis may be made with the aid of direct fluorescence antibody for HSV and VZV from vesicular lesions or PCR from CSF or visceral tissue samples. Treatment for disseminated infections involves intravenous acyclovir, while less severe infection can be treated with oral acyclovir, valacyclovir, or famciclovir [6].

EBV is a ubiquitous herpesvirus and it maintains latency. Under profound immunosuppression, profound impairment of the cytotoxic T-cell response results in failure to maintain latency, leading to uncontrolled EBV-driven B-cell proliferation and posttransplant lymphoproliferative disease (PTLD), which is why this virus is clinically important. EBV infection (and relapses in the absence of antiviral immunity) causes a mononucleosis-type syndrome, generally presenting as a lymphocytosis with or without lymphadenopathy or pharyngitis [7]. Meningitis, hepatitis, and pancreatitis may be also observed. Compared with lymphoma in the general population, PTLD has increased extranodal involvement, poorer response to conventional therapies, and poorer outcomes. Combinations of anti-B cell therapy (anti-CD20; Rituximab), chemotherapy (CHOP), irradiation (for central nervous system disease), and/or adoptive immunotherapy with stimulated $\mathrm{T}$ cells have been used [8]. Cessation of immune suppression may precipitate allograft loss.

The polyomaviruses are DNA viruses, and three species are known to infect man: BK virus, JC virus, and a simian virus, SV40. Initial infection is usually asymptomatic and probably occurs via the respiratory route or as a blood-borne infection. Known risk factors in the transplant recipient include multiple rejection episodes and a seropositive donor and/or recipient. As the use of potent immunosuppressive agents such as tacrolimus, mycophenolate mofetil, and sirolimus has increased, so have reports of polyomavirus nephropathy (PVAN) [9]. PVAN is characterized by mononuclear cell interstitial infiltrates and tubulitis, which can be confused with acute cellular rejection. Recognition is critical since the correct therapy is reduction, rather than intensification, of immunosuppression. Viral shedding in the urine and serologic activation are not usually associated with symptoms. Urine cytology is technically the simplest method for monitoring polyomavirus infection after transplantation. Therapeutic strategies for polyomavirus continue to evolve. No prospective studies have been done to determine whether patients with asymptomatic viremia or viruria benefit from specific therapeutic intervention, but there is a general consensus that optimal therapy of patients with PVAN is reduction in immunosuppression.

HHV-6 and HHV-7 are increasingly recognized as pathogens in transplant recipients. HHV-6 and HHV-7 are homologous to CMV. HHV- 6 has been associated with fever, rash, encephalitis, hepatitis, myelosuppression, and interstitial pneumonitis [8,10]. HHV-6 and CMV are significantly and independently associated with biopsy-proven graft rejection. Diagnoses of HHV-6 and HHV7 infections are made by qualitative and quantitative molecular assays, by tissue immunohistochemistry or peripheral blood mononuclear cell culture. Treatment includes reducing immunosuppression and antiviral agents. In vitro, HHV-6 and HHV-7 are most susceptible to cidofovir, with ganciclovir. HHV-8 is associated with Kaposi sarcoma (KS) and primary effusion lymphoma in immunocompromised individuals from endemic regions. Seroconversion is common at the time of solid-organ transplantation. Transplantation-associated KS occurs in 0.2 to $5 \%$ of renal transplant recipients, varying by ethnic group and immunosuppressive regimen [11]. In addition, HHV-8 may be associated with some cases of EBV-negative PTLD [10,11]. Treatment usually involves reducing the immunosuppressive regimen and treating with chemotherapy or foscarnet [11].

HTLV-1 is endemic in parts of Asia and can progress to HTLVI-associated myelopathy or to adult T cell leukemia/lymphoma. HTLV-II is similar to HTLV-I serologically but is less clearly associated with disease. Use of organs 
from such donors is avoided because transplant recipients can experience more aggressive disease. Optimal therapy (antiretrovirals) remains to be defined $[8,12]$.

Patients with chronic renal failure, notably those receiving hemodialysis, may be at increased risk for Hepatitis B. Approximately 2 to 10 percent of patients with a history of Hepatitis B before transplantation will reactivate post transplant. Previously, patients who were Hepatitis B surface antigen positive were not considered to be acceptable candidates for transplantation; however the more recent availability of effective antiviral agents has allowed consideration of the recipient with chronic Hepatitis $B$ who has cleared viremia $[1,13]$. Treatment of chronic hepatitis $B$ includes reduction of immunosuppression whenever possible. Optimal therapy should include the use of at least one antiviral active against Hepatitis B; currently lamivudine is most commonly used. Alternative antivirals with activity against Hepatitis B include IFN, adefovir, entecavir and telbivudine. Because of the potential for renal toxicity, these potentially more effective agents are not commonly used in renal transplant recipients.

$\mathrm{HCV}$ infection is associated with increased morbidity and/or mortality in renal transplant recipients. In a retrospective study on the impact of hepatitis $C$ virus infection on kidney transplant patients, HCV antibody carriers had a poor survival rate (because of liver dysfunction) in the second decade compared with the noninfected group [8]. However, in one of our study performed to investigate the impact of HCV infection on the long-term survival of renal transplant recipients, for an eight-year follow-up period, we concluded that chronic $\mathrm{HCV}$ infection before transplantation did not have a significant impact on graft survival or mortality compared with noninfected patients [14]. In this study, outcomes and survivals among 325 patients who received renal allografts were compared between those known to have pretransplantation HCV infection (Group I, HCV + group, $\mathrm{n}=33$ ) versus a matched cohort of those without this infection (Group II, HCV-control group, $n=33$ ). Allograft performance, liver function, cholesterol, and glucose levels were determined both at transplantation and at a mean of postgrafting year 8. Thirty-three patients (10.15\%) were positive for HCV antibody. The mean survival rates were similar in Groups I and II (96.6\% and, $100 \%$, respectively). Although the allograft survival rate was lower in Group I (84.8\% vs. 90.9\%), the rejection rate among the HCV-group was $6 \%$; only 1 patient died of hepatic failure. In spite of a significant rise in both total and direct bilirubin values $(P<0.01)$ in both groups, we failed to observe an adverse effect on graft survival. A significant rise in the fasting glucose level was seen in both $\mathrm{HCV}+$ and $\mathrm{HCV}$ - patients. IFN treatment of $\mathrm{HCV}$ infection carries a risk for graft rejection in renal transplant recipients and such use needs to be very carefully evaluated. Ribavirin monotherapy can be used as an alternative treatment with careful follow-up.
Recent studies confirm that outcome of renal transplantation in adequately selected HIV-infected patients receiving kidneys from HIV-negative donors is similar to that of HIV-negative renal transplant recipients [1]. Main challenges in the clinical management of HIV-infected $\mathrm{RT}$ recipients are the pharmacologic interactions between immunosuppressive agents and some classes of antiretroviral drugs and a higher rate of acute rejection in comparison with HIV-negative recipients. HIV-infected patients receiving renal transplants may be at higher risk of acute rejection (up to $25 \%$ ) and the optimal management of immunosuppression in HIV-infected individuals remains unknown. Treatment of rejection with cytolytic agents such as thymoglobulin may result in prolonged depression of CD4 counts and significant infection-related morbidity.

HPV infections can cause significant disease in renal transplant recipients, including oral, skin, genital, and rectal lesions ranging from warts and dysplasia to malignancy. Renal transplant recipients have a significantly increased risk of ano-genital cancer and nonmelanoma skin cancer [8]. Treatment requires decreasing immunosuppression, topical, or surgical treatment. Topical immunotherapies such as imiquimod should be used with caution.

Transplant recipients are at higher risk than the general population for meningoencephalitis after exposure to WNV in endemic regions. Virus from their donors have increased morbidity and mortality [1,8]. Recipients who acquire West Nile later in the transplant course have more variable outcomes. To prevent infection, seasonal screening should be considered for donors before transplant by serologic and/or nucleic acid testing. Treatment for West Nile in transplant recipients has not been standardized but should include a reduction in immunosuppression along with supportive care.

Important community-acquired respiratory virus infections in the immunocompromised host include influenza virus types $A$ and $B$; respiratory syncytial virus; parainfluenza viruses 1, 2, and 3; adenoviruses; and possibly, rhinoviruses [5]. Transmission is via contact for all viruses except influenza, which is transmitted by respiratory droplets. Common respiratory virus syndromes include the common cold, upper respiratory tract infection, pharyngitis, laryngitis, tracheobronchitis, influenza-like syndromes, bronchiolitis, and pneumonia. In the immunocompromised host, viral shedding may persist for months vs. days in the immunocompetent host. Treatment of respiratory viral infections involves supportive care and, in some cases, the use of antiviral medications. Influenza can be treated with oseltamivir or zanamavir, which will treat both influenza A and B. Ribavirin is approved to treat lower respiratory infection with respiratory syncytial virus. Influenza vaccine should be administered pretransplant and every year after transplant, although administration should not be 
given in the early post transplant period because of especially reduced vaccine responses.

Rabies transmission has been reported with transplantation of organs from deceased donors [8]. The diagnosis was confirmed in four recipients of transplanted organs and in their common donor, who was found subsequently to have serologic evidence of rabies infection. The recipients had encephalitis of unknown cause after transplantation. Rabies is acute encephalitis caused by neurotropic virus with an incubation time generally of several weeks to months. Rabies postexposure prophylaxis is highly effective in preventing rabies when administered before onset of clinical signs.

\section{References}

1. Weikert BC, Blumberg EA (2008) Viral infection after renal transplantation: Surveillance and management. Clin J Am Soc Nephrol 3: S76-S86.

2. Rostaing L, Wéclawiak H, Mengelle C, Kamar N (2011) Viral infections after kidney transplantation. Minerva Urol Nefrol 63: 59-71.

3. Cordero E, Casasola C, Ecarma R, Danguilan R (2012) Cytomegalovirus disease in kidney transplant recipients: Incidence, clinical profile, and risk factors. Transplant Proc 44: 694-700.

4. Arasaratnam RJ (2015) Lower-dose valganciclovir for the prevention of cytomegalovirus after solid organ transplantation: An important tradeoff. Transpl Infect Dis 17: 623-624.

5. Green M, Avery R, Preiksaitis J (2004) Guidelines for the prevention and management of infectious complications of solid organ transplantation. Am J Transplant 4: 160-163.

6. Cowan FM, Copas A, Johnson AM, Ashley R, Corey L, et al. (2002) Herpes simplex type 1 infection: A sexually transmitted infection of adolescence? Sex Transm Infect 78: $346-348$

7. Roschewski M (2012) EBV-associated lymphomas in adults. Best Pract Res Clin Haematol 25: 75-89.

8. Kotton CN, Fishman JA (2005) Viral infection in the renal transplant recipient. J Am Soc Nephrol 16: 1758-1774.

9. Costa C, Cavallo R (2012) Polyomavirus-associated nephropathy. World J Transplant 2: 84-94.

10. Rossi C, Delforge ML, Jacobs F, Wissing M, Pradier O, et al. (2001) Fatal primary infection due to human herpesvirus 6 variant $A$ in a renal transplant recipient. Transplantation 71: 288-292.

11. Penn I (1997) Kaposi's sarcoma in transplant recipients. Transplantation 64: 669-673.

12. Toro C, Rodes B, Poveda E, Soriano V (2003) Rapid development of subacute myelopathy in three organ transplant recipients after transmission of human T-cell lymphotropic virus type I from a single donor. Transplantation 75: 102104.

13. Karuthu S, Blumberg EA (2012) Common infections in kidney transplant recipients. Clin J Am Soc Nephrol 7: 20582070.

14. Unal E, Turkmen F, Sevinc C, Kiziler AR, Aydemir B, et al. (2006) Outcome of renal allograft in Turkish patients with pretransplantation hepatitis $\mathrm{C}$ virus infection. Transplant Proc 38: 3448-3450. 